\title{
A 3D numerical model for turbidity currents
}

\author{
GIOVANNI CANNATA, LUCA BARSI, MARCO TAMBURRINO \\ Department of Civil, Constructional and Environmental Engineering \\ "Sapienza" University of Rome \\ Via Eudossiana 18 - 00184, Roma \\ ITALY \\ giovanni.cannata@uniroma1.it
}

\begin{abstract}
A numerical model that solves two-phase flow motion equations to reproduce turbidity currents that occur in reservoirs, is proposed. Three formalizations of the two-phase flow motion equations are presented: the first one can be adopted for high concentration values; the second one is valid under the hypothesis of diluted concentrations; the third one is based on the assumption that the particles are in translational equilibrium with the fluid flow. The proposed numerical model solves the latter formalization of two-phase flow motion equations, in order to simulate turbidity currents. The motion equations are presented in an integral form in time-dependent curvilinear coordinates, with the vertical coordinate that varies in order to follow the free surface movements. The proposed numerical model is validated against experimental data and is applied to a practical engineering case study of a reservoir, in order to evaluate the possibility of the formation of turbidity currents.
\end{abstract}

Key-Words: - turbidity current, two-phase flow, free surface flow, numerical model, time-varying coordinates

Received: August 7, 2019. Revised: January 6, 2020. Re-revised: January 17, 2020.

Accepted: January 23, 2020. Published: January 27, 2020.

\section{Introduction}

The motion equations of a two-phase flow with a mutual interaction between the phases are commonly used to represent many engineering problems. In this context, Siddiqui et al. [1] used the semi-analytic Homotopy Analysis Method to solve the two-phase flow motion equations for a CouettePoiseuille flow. In Mitkova et al. [2], the layer thickness variation in two-phase flow of a thirdgrade fluid, is investigated. Abood et al. [3-4] carried out a numerical-experimental study of air-oil [3] and water-oil [4] two-phase flows, for the simulation of the stream patterns in vertical and horizontal pipes. Abdulwahid et al. [5] made a numerical investigation on two-phase air-water flows in pipes with VOF homogeneous model through unsteady state turbulent flow employed to study the effect of holdup, void fraction and liquid film thickness on the pressure drop through pipes.

Gravity currents consist in a two-phase flow that is driven by the density difference between the phases. These phenomena have been investigated extensively in the past years, numerically, experimentally and analytically. Ungarish [6] presented an analytical analysis of a steady-state gravity current that propagates into an ambient of motionless fluid in an open channel of general nonrectangular cross-section at high Reynolds number. Longo et al. [7] carried out a theoretical and experimental study of non-Boussinesq inertial (high Reynolds number flows) gravity currents flowing in rectangular cross-sections, produced by lockrelease. Hogg et al. [8] investigated theoretically on gravitationally driven motion arising from a sustained constant source of dense fluid in a horizontal channel, using shallow-layer models and direct numerical simulations of the Navier-Stokes equations. Salinas et al. [9] used a 3D DNS model to investigate on the flow of a gravity current of a dense fluid, released from rest from a rectangular lock, into an ambient fluid with a density lower than the one of the dense fluid. See [10] for a detailed review about gravity currents.

Turbidity currents can be considered as particular gravity currents, in which solid particles represent one of the phases. In recent years, Espath et al. [11] and Cantero et al. [12] carried out numerical studies on turbidity currents, based on direct numerical simulations. Turbidity currents in reservoirs (which are created by artificial barrages on water courses), that can occur when the flow has the capacity to transport large quantities of solid materials, can be represented as a free-surface two-phase flow (with a fluid phase representing the water and a solid phase representing the solid particles). In the representation of the aforementioned phenomenon, a two-way coupling between fluid and solid phases, must be used. 
In the context of the numerical simulation of free-surface flows, many models solve the depthaveraged motion equations, as Nonlinear Shallow Water Equations [13] or Boussinesq Equations [1415], and the depth-averaged concentration equation. In these models, the velocity and the concentration fields are averaged over the water depth. In order to have a proper representation of a two-way coupled two-phase flow, is important to have a prediction of the vertical distribution of the flow variables.

In order to take into account the threedimensional aspects of the fluid flows, many recent models numerically solve the three-dimensional motion equations [16-18]. In the context of threedimensional models for free surface flows, the correct assignment of pressure and kinematic boundary condition at the free-surface is one of the most challenging issues. A recent class of models [19-20] solves this issue by using the so-called $\sigma$ coordinates transformation. By doing so, the zeropressure and kinematic boundary conditions at the free surface can be assigned precisely.

In the formalization of the two-phase flow equations, some hypotheses can be adopted to simplify the representation of the physical phenomena: a) there is a thermal and dynamic equilibrium between the phases; $b$ ) the solid phase can be considered as a homogeneous fluid, so that the mass and momentum conservation principles are valid for it; c) the solid particles are spherical in shape. Under these hypotheses, mass and momentum conservation equations can be defined for each phase (solid and fluid). There is a two-way coupling between the set of equations of the fluid phase and the set of equations of the solid phase, due to the terms that arise in both sets of the equations. These terms are: the forces resulting from the interaction between the two phases and the volume fraction of the solid phase (the ratio between the macroscopic density of the solid phase and the microscopic density of the single particle). In this work, three formulations are presented, which introduce subsequent simplifications. The most general formalization is valid for high solid phase concentrations. In this formalization, the fluid flow is not isochoric, because in the continuity equation of the fluid phase, the presence of the volume fraction of the solid phase term does not allow the fluid velocity vector field to be divergence free.

The second formalization introduces the hypothesis of diluted concentration. The volume fraction of the solid phase can therefore be neglected in the continuity equation and in all the terms of the momentum balance equation of the fluid phase, except from the body force term; the two-way coupling between the phases is guaranteed by the presence of the volume fraction of the solid phase in the body force term of the momentum balance equation of the fluid phase. Furthermore, being the suspension diluted, the solid particles are sufficiently far one from each other, to let us assume that the interaction stresses between the solid particles are negligible. In the formalization of the two-phase flow motion equations for a diluted suspension, the two sets of equations are still twoway coupled (through the interaction force between the two phases and the volume fraction of the solid phase), but the fluid flow is isochoric.

In the third formalization of the motion equations, the so-called linearization hypothesis is introduced: when the ratio between the particle translational relaxation time and the characteristic time of the fluid flow (i.e. the Stokes number $S k$ ) is sufficiently low $\left(S k<10^{-1}\right)$, it is possible to make the assumption that the particles are in translational equilibrium with the fluid flow. Under this hypothesis, the horizontal velocity components of the solid phase are equal to the horizontal velocity components of the fluid phase, while the vertical velocity component of the solid phase is equal to the sum of the vertical velocity component of the fluid phase and the particle settling velocity. The momentum equation of the solid phase and the momentum equation of the liquid phase can be combined, in order to obtain a single momentum equation. The other two equations that are present in this formalization are the mass conservation equation for the fluid phase, and the particle concentration equation, derived from the mass conservation equation for the solid phase. Formulations of motion equations that introduce the linearization hypothesis are extensively used in the context of numerical simulation of turbidity currents (see for example [11] and [12]).

In this work, the aforementioned three different formalizations of the two-phase flow motion equations, are expressed in integral form, in terms of Cartesian based variables, on a time-dependent domain. A numerical model for the simulation of the turbidity currents, that solves the two-phase flow three-dimensional motion equations in integral form under the linearization hypothesis, is proposed. The motion equations, expressed in terms of Cartesian based variables, are transformed in a time-varying 
coordinate system and are numerically integrated on a time-varying boundary conforming grid. Following the procedure shown in [17], the aforementioned equations are solved on a coordinate system in which the vertical coordinate varies in time in order to follow the free-surface movement. The solution advances in time by using a Strong Stability Preserving Runge-Kutta (SSPRK) fractional step method; at each stage, a divergencefree velocity field is obtained by applying a pressure correction formulation.

The proposed model is validated against experimental data presented by [21]. Furthermore, the proposed model is applied to a practical engineering case study of a reservoir. In fact, during floodings, large quantities of solid material are transported into reservoirs and then are blocked by the artificial barrages. A strategy to solve this issue consists in the opening of the bottom outlets of the dam during floodings, which can lead to the formation of turbidity currents that are able to transport downstream the solid material. The aim of this investigation is to verify if the opening the bottom outlet of the dam causes the formation a turbidity current, that is able discharge downstream the solid material which reaches the reservoir during a flooding, in order to control the silting processes in the reservoir.

The paper is structured as follows: in Section 2, we present the different formulation of the twophase flow motion equations and the numerical model; in Section 3, we show the results of a validation of the proposed model against several experimental tests and we present a practical engineering case study of a reservoir; in Section 4, we present the conclusion of our work.

\section{Equations and model}

\subsection{Governing Equations}

The motion equations of a two-phase flow with mutual interaction between phases can be used to simulate turbidity current. In order to simplify the representation of the physical phenomenon, three hypotheses can be adopted:

1. thermal and dynamic equilibrium between phases;

2. solid phase as an homogeneous fluid, so that the mass and momentum conservation principles are valid for it;

3. spherical solid particles with diameter $d$.
Let $\breve{\rho}_{S}$ be the microscopic density of the single solid particle and $\rho_{S}$ be the macroscopic density of the solid phase; $a=\rho_{S} / \breve{\rho}_{S}$ is the volume fraction of the solid phase. Let $\rho$ be the microscopic density of the fluid phase.

Let us consider a control volume $\Delta V(t)$, with surface $\Delta A(t)$, which moves with a velocity $\vec{v}$ different from the fluid velocity $\vec{u}$. In a Cartesian system of reference $\left(x_{1}, x_{2}, x_{3}\right), \vec{u}$ and $\vec{v}$ components are respectively $\left(u_{1}, u_{2}, u_{3}\right)$ and $\left(v_{1}, v_{2}, v_{3}\right)$. The integral form of the mass conservation equation of the fluid phase over the control volume $\Delta V(t)$ is:

$$
\begin{gathered}
\rho \frac{d}{d t} \int_{\Delta V(t)}(1-a) d V \\
+\rho \int_{\Delta A(t)}(1-a)(\vec{u}-\vec{v}) \cdot \hat{n} d A=0
\end{gathered}
$$

where $\hat{n}$ is the unit outward normal vector. The integral form of the momentum equation of the fluid phase over the control volume $\Delta V(t)$ is:

$$
\begin{gathered}
\rho \frac{d}{d t} \int_{\Delta V(t)}(1-a) \vec{u} d V \\
+\rho \int_{\Delta A(t)}(1-a)[\vec{u} \otimes(\vec{u}-\vec{v})] \cdot \hat{n} d A \\
=\rho \frac{d}{d t} \int_{\Delta V(t)}(1-a) \vec{f} d V+\int_{\Delta A(t)} \mathbf{T} \cdot \hat{n} d A \\
-\int_{\Delta V(t)} \vec{F}_{P} d V
\end{gathered}
$$

where the symbol $\otimes$ represents the outer product, $\vec{f}$ is the external body force vector per unit mass, $\mathbf{T}$ is the viscous stress tensor and $\vec{F}_{P}$ is the force per unit volume resulting from the interaction between the two phases, which is given by:

$$
\vec{F}_{P}=a \frac{18 \mu}{d^{2}}\left(\vec{u}-\vec{u}_{S}\right)-a \nabla p
$$

where $\mu$ and $p$ are the dynamic viscosity and the pressure of the fluid phase, respectively, and $\vec{u}_{S}$ is the velocity vector of the solid phase. The first term of eqn. (3) is the viscous resistance force according to Stokes law, while the second term is due to the pressure gradient, in the fluid surrounding the particle, caused by the acceleration of the fluid. 
The integral form of the mass conservation equation of the solid phase over the control volume $\Delta V(t)$ is:

$$
\begin{gathered}
\breve{\rho}_{S} \frac{d}{d t} \int_{\Delta V(t)} a d V \\
+\breve{\rho}_{S} \int_{\Delta A(t)} a\left(\vec{u}_{S}-\vec{v}\right) \cdot \hat{n} d A=0
\end{gathered}
$$

The integral form of the momentum equation of the solid phase over the control volume $\Delta V(t)$ is:

$$
\begin{gathered}
\breve{\rho}_{S} \frac{d}{d t} \int_{\Delta V(t)} a \vec{u}_{S} d V \\
+\breve{\rho}_{S} \int_{\Delta A(t)} a\left[\vec{u}_{S} \otimes\left(\vec{u}_{S}-\vec{v}\right)\right] \cdot \hat{n} d A \\
=\breve{\rho}_{S} \frac{d}{d t} \int_{\Delta V(t)} a \vec{f} d V+\int_{\Delta A(t)} \mathbf{T}_{S} \cdot \hat{n} d A \\
+\int_{\Delta V(t)} \vec{F}_{P} d V
\end{gathered}
$$

where $\mathbf{T}_{S}$ is the solid phase stress tensor that takes into account the effects of the interactions between two or more solid particles.

Let $\left(\xi_{1}, \xi_{2}, \xi_{3}\right)$ be a system of curvilinear coordinates, the transformation from Cartesian coordinates $\left(x_{1}, x_{2}, x_{3}\right)$ to the generalized curvilinear coordinates $\left(\xi_{1}, \xi_{2}, \xi_{3}\right)$ is:

$$
\begin{aligned}
& \xi_{1}=\xi_{1}\left(x_{1}, x_{2}, x_{3}\right) \\
& \xi_{2}=\xi_{2}\left(x_{1}, x_{2}, x_{3}\right) \\
& \xi_{3}=\xi_{3}\left(x_{1}, x_{2}, x_{3}\right)
\end{aligned}
$$

Let $\vec{c}_{(l)}=\partial \vec{x} / \partial \xi_{l}$ be the covariant base vectors and $\vec{c}^{(l)}=\partial \xi_{l} / \partial \vec{x}$ the contravariant base vectors. The metric tensor and its inverse are defined by $c_{l m}=\vec{c}_{(l)} \cdot \vec{c}_{(m)} \quad$ and $\quad c^{l m}=\vec{c}^{(l)} \cdot \vec{c}^{(m)}$, with $(l, m=1,2,3)$. The Jacobian of the transformation is given by [22-23]:

$$
\sqrt{c}=\sqrt{\operatorname{det}\left(c_{l m}\right)}
$$

$\Delta V(t)$ is considered as a volume element defined by surface elements bounded by curves lying on the coordinate lines. We define the volume element in the physical space as $\Delta V(t)=\sqrt{c} \Delta \xi_{1} \Delta \xi_{2} \Delta \xi_{3}$ and the volume element in the transformed space as $\Delta V^{*}=\Delta \xi_{1} \Delta \xi_{2} \Delta \xi_{3}$. It is possible to see that $\Delta V(t)$ is time dependent, while $\Delta V^{*}$ is not. Analogously, the surface element in the physical space as $\Delta A_{\alpha \beta}(t)$ is time-dependent, while the surface element in the transformed space $\Delta A_{\alpha \beta}^{*}$ is not.

In a generalized curvilinear coordinate system, eqns. (1) and (2) become, respectively:

$$
\begin{aligned}
& \rho \frac{d}{d t} \int_{\Delta V^{*}}(1-a) \sqrt{c} d \xi_{1} d \xi_{2} d \xi_{3}= \\
& -\rho \sum_{\alpha=1}^{3}\left[\int_{\Delta A_{\beta \gamma}^{*+}}(1-a)(\vec{u}-\vec{v})\right. \\
& \cdot \vec{c}^{(\alpha)} \sqrt{c} d \xi_{\beta} d \xi_{\gamma} \\
& \left.-\int_{\Delta A_{\beta \gamma}^{*-}}(1-a)(\vec{u}-\vec{v}) \cdot \vec{c}^{(\alpha)} \sqrt{g} d \xi_{\beta} d \xi_{\gamma}\right] \\
& \rho \frac{d}{d t} \int_{\Delta V^{*}}(1-a) \vec{u} \sqrt{c} d \xi_{1} d \xi_{2} d \xi_{3}= \\
& -\rho \sum_{\alpha=1}^{3}\left\{\int_{\Delta A_{\beta \gamma}^{*+}}(1-a)[\vec{u} \otimes(\vec{u}-\vec{v})]\right. \\
& \cdot \vec{c}^{(\alpha)} \sqrt{c} d \xi_{\beta} d \xi_{\gamma} \\
& -\int_{\Delta A_{\beta \gamma}^{*-}}(1-a)[\vec{u} \otimes(\vec{u}-\vec{v})] \\
& \left.\cdot \vec{c}^{(\alpha)} \sqrt{c} d \xi_{\beta} d \xi_{\gamma}\right\} \\
& +\sum_{\alpha=1}^{3}\left[\int_{\Delta A_{\beta \gamma}^{*+}} \boldsymbol{T} \cdot \vec{c}^{(\alpha)} \sqrt{c} d \xi_{\beta} d \xi_{\gamma}\right. \\
& \left.-\int_{\Delta A_{\beta \gamma}^{*-}} \boldsymbol{T} \cdot \vec{c}^{(\alpha)} \sqrt{c} d \xi_{\beta} d \xi_{\gamma}\right] \\
& +\rho \int_{\Delta V^{*}}(1-a) \vec{f} \sqrt{c} d \xi_{1} d \xi_{2} d \xi_{3} \\
& -\int_{\Delta V^{*}} \vec{F}_{P} \sqrt{c} d \xi_{1} d \xi_{2} d \xi_{3}
\end{aligned}
$$

In eqns. (8), (9) and hereinafter, $\Delta A_{\beta \gamma}^{*+}$ and $\Delta A_{\beta \gamma}^{*-}$ are the contour surfaces of the volume $\Delta V^{*}$ on which $\xi_{\alpha}$ is constant and which are located at the larger and the smaller value of $\xi_{\alpha}$, respectively (the coefficients $\alpha, \beta, \gamma=1,2,3$ are cyclic). In a generalized curvilinear coordinate system, eqns. (4) and (5) become, respectively:

$$
\begin{gathered}
\breve{\rho}_{S} \frac{d}{d t} \int_{\Delta V^{*}} a \sqrt{c} d \xi_{1} d \xi_{2} d \xi_{3}= \\
-\breve{\rho}_{S} \sum_{\alpha=1}^{3}\left[\int_{\Delta A_{\beta \gamma}^{*+}} a\left(\vec{u}_{S}-\vec{v}\right) \cdot \vec{c}^{(\alpha)} \sqrt{c} d \xi_{\beta} d \xi_{\gamma}\right.
\end{gathered}
$$




$$
\begin{gathered}
\left.-\int_{\Delta A_{\beta \gamma}^{*-}} a\left(\vec{u}_{S}-\vec{v}\right) \cdot \vec{c}^{(\alpha)} \sqrt{c} d \xi_{\beta} d \xi_{\gamma}\right] \\
\breve{\rho}_{S} \frac{d}{d t} \int_{\Delta V^{*}} a \vec{u}_{S} \sqrt{c} d \xi_{1} d \xi_{2} d \xi_{3}= \\
-\breve{\rho}_{S} \sum_{\alpha=1}^{3}\left\{\int_{\Delta A_{\beta \gamma}^{*+}} a\left[\vec{u}_{S} \otimes\left(\vec{u}_{S}-\vec{v}\right)\right]\right. \\
\left.-\int_{\Delta A_{\beta \gamma}^{*-}} a\left[\vec{u}_{S} \otimes\left(\vec{u}_{S}-\vec{v}\right)\right] \cdot \vec{c}^{(\alpha)} d \xi_{\beta} d \xi_{\gamma} d \xi_{\beta} d \xi_{\gamma}\right\} \\
+\sum_{\alpha=1}^{3}\left[\int_{\Delta A_{\beta \gamma}^{*+}} \mathbf{T}_{S} \cdot \vec{c}^{(\alpha)} \sqrt{c} d \xi_{\beta} d \xi_{\gamma}\right. \\
\left.-\int_{\Delta A_{\beta \gamma}^{*-}} \mathbf{T}_{S} \cdot \vec{c}^{(\alpha)} \sqrt{c} d \xi_{\beta} d \xi_{\gamma}\right] \\
+\breve{\rho}_{S} \int_{\Delta V^{*}} a \vec{f} \sqrt{c} d \xi_{1} d \xi_{2} d \xi_{3} \\
+\int_{\Delta V^{*}} \vec{F}_{P} \sqrt{c} d \xi_{1} d \xi_{2} d \xi_{3}
\end{gathered}
$$

Eqns. (8), (9), (10) and (11) represent the general formalization of the two-phase flow equations in integral form. In eqn. (8), the presence of the volume fraction term $a$ does not allow the fluid velocity vector field to be divergence free.

If the suspension is sufficiently diluted, it is possible to obtain a simplified formulation for the two-phase flow equations. The solid phase stress tensor $\mathbf{T}_{S}$ components are inversely proportional to the ratio of the distance between particles to their diameter; therefore, $\mathbf{T}_{S}$ can be neglected for diluted suspensions. Furthermore, the volume fraction $a$ can be neglected in the continuity equation and in all the terms of the momentum balance equation of the fluid phase, except from the body force term. With these assumptions, eqns. (8), (9), (10) and (11) become, respectively:

$$
\begin{gathered}
\rho \frac{d}{d t} \int_{\Delta V^{*}} \sqrt{c} d \xi_{1} d \xi_{2} d \xi_{3}= \\
-\rho \sum_{\alpha=1}^{3}\left[\int_{\Delta A_{\beta \gamma}^{*+}}(\vec{u}-\vec{v}) \cdot \vec{c}^{(\alpha)} \sqrt{c} d \xi_{\beta} d \xi_{\gamma}\right. \\
\left.-\int_{\Delta A_{\beta \gamma}^{*-}}(\vec{u}-\vec{v}) \cdot \vec{c}^{(\alpha)} \sqrt{c} d \xi_{\beta} d \xi_{\gamma}\right]
\end{gathered}
$$

$$
\begin{aligned}
& \rho \frac{d}{d t} \int_{\Delta V^{*}} \vec{u} \sqrt{c} d \xi_{1} d \xi_{2} d \xi_{3}= \\
& -\rho \sum_{\alpha=1}^{3}\left\{\int_{\Delta A_{\beta \gamma}^{*+}}[\vec{u} \otimes(\vec{u}-\vec{v})] \cdot \vec{c}^{(\alpha)} \sqrt{c} d \xi_{\beta} d \xi_{\gamma}\right. \\
& \left.-\int_{\Delta A_{\beta \gamma}^{*-}}[\vec{u} \otimes(\vec{u}-\vec{v})] \cdot \vec{c}^{(\alpha)} \sqrt{c} d \xi_{\beta} d \xi_{\gamma}\right\} \\
& +\sum_{\alpha=1}^{3}\left[\int_{\Delta A_{\beta \gamma}^{*+}} \mathbf{T} \cdot \vec{c}^{(\alpha)} \sqrt{c} d \xi_{\beta} d \xi_{\gamma}\right. \\
& \left.-\int_{\Delta A_{\beta \gamma}^{*-}} \mathbf{T} \cdot \vec{c}^{(\alpha)} \sqrt{c} d \xi_{\beta} d \xi_{\gamma}\right] \\
& +\rho \int_{\Delta V^{*}}(1-a) \vec{f} \sqrt{c} d \xi_{1} d \xi_{2} d \xi_{3} \\
& -\int_{\Delta V^{*}} \vec{F}_{P} \sqrt{c} d \xi_{1} d \xi_{2} d \xi_{3} \\
& \breve{\rho}_{S} \frac{d}{d t} \int_{\Delta V^{*}} a \sqrt{c} d \xi_{1} d \xi_{2} d \xi_{3}= \\
& -\breve{\rho}_{S} \sum_{\alpha=1}^{3}\left[\int_{\Delta A_{\beta \gamma}^{*+}} a\left(\vec{u}_{S}-\vec{v}\right) \cdot \vec{c}^{(\alpha)} \sqrt{c} d \xi_{\beta} d \xi_{\gamma}\right. \\
& \left.-\int_{\Delta A_{\beta \gamma}^{*-}} a\left(\vec{u}_{S}-\vec{v}\right) \cdot \vec{c}^{(\alpha)} \sqrt{c} d \xi_{\beta} d \xi_{\gamma}\right] \\
& \breve{\rho}_{S} \frac{d}{d t} \int_{\Delta V^{*}} a \vec{u}_{S} \sqrt{c} d \xi_{1} d \xi_{2} d \xi_{3}= \\
& -\breve{\rho}_{S} \sum_{\alpha=1}^{3}\left\{\int_{\Delta A_{\beta \gamma}^{*+}} a\left[\vec{u}_{S} \otimes\left(\vec{u}_{S}-\vec{v}\right)\right]\right. \\
& \cdot \vec{c}^{(\alpha)} \sqrt{c} d \xi_{\beta} d \xi_{\gamma} \\
& \left.-\int_{\Delta A_{\beta \gamma}^{*-}} a\left[\vec{u}_{S} \otimes\left(\vec{u}_{S}-\vec{v}\right)\right] \cdot \vec{c}^{(\alpha)} \sqrt{c} d \xi_{\beta} d \xi_{\gamma}\right\} \\
& +\breve{\rho}_{S} \int_{\Delta V^{*}} a \vec{f} \sqrt{c} d \xi_{1} d \xi_{2} d \xi_{3} \\
& +\int_{\Delta V^{*}} \vec{F}_{P} \sqrt{c} d \xi_{1} d \xi_{2} d \xi_{3}
\end{aligned}
$$

Eqns. (12), (13), (14) and (15) represent the formalization of the two-phase flow motion equations in integral form for a diluted suspension. Is to be noted that fluid flow is assumed to be isochoric, as in eqn. (12) the volume fraction $a$ is neglected.

Let us introduce the loading ratio of the flow and the Stokes number, that are respectively:

$$
\beta=\frac{\breve{\rho}_{S}}{\rho}
$$




$$
S k=\frac{\tau_{p}}{\tau_{f}}
$$

$\tau_{p}$ is the particle translational relaxation time and $\tau_{f}$ is the characteristic time of the fluid flow. The translational relaxation time can be expressed as:

$$
\tau_{p}=\frac{\breve{\rho}_{S} d^{2}}{18 \mu f_{d}}
$$

where $f_{d}$ is the drag coefficient:

$$
f_{d}=1+\frac{1}{6}\left(R_{p}\right)^{2 / 3}
$$

with the particle Reynolds number $R_{p}$ equal to:

$$
R_{p}=\frac{\rho d\left|\vec{u}-\vec{u}_{S}\right|}{\mu}
$$

The characteristic time of the fluid flow can be expressed as

$$
\tau_{f}=\frac{L}{U}
$$

where $L$ and $U$ are the flow field characteristic length and the flow characteristic velocity, respectively. The Stokes number indicates how rapidly the particle is able to follow the variations in the fluid velocity and it is proportional to the translational non-equilibrium of the particles. Small values of $S k$ means a small difference between the particle and the fluid velocity, and large coupling effect. The value of the Stokes number indicates whether it is possible to consider the particles in equilibrium with the fluid flow: if $S k<10^{-1}$, the hypothesis of particles in equilibrium with the fluid flow can be assumed, so the particle velocity field can be assumed as a superimposition of the fluid velocity field and the particle settling velocity field; if $S k>10^{-1}$, the nonequilibrium effects are not negligible.

The loading ratio $\beta$ is related to the coupling effect. For small values of $\beta$, the fluid phase is not dependent to the solid phase, resulting in a one-way coupling between the set of equations of the fluid phase and the set of equations of the solid phase.
Large values of $\beta$ lead to a two-way coupling between the two sets of equations, which have to be solved simultaneously.

In the case of turbidity currents, the values of $S k$ are small, so the the particles can be considered in equilibrium with the fluid phase. Thus, the linearization hypothesis can be introduced: the solid phase velocity field is obtained by superimposing the fluid flow velocity field and the solid particle settling velocity field. On the other hand, turbidity currents have large values of $\beta$, so the effects of the solid particles on the fluid flow cannot be neglected.

Let be $H\left(x_{1}, x_{2}, t\right)=h\left(x_{1}, x_{2}\right)+\eta\left(x_{1}, x_{2}, t\right)$, where $h$ is the still water depth. Let us assume that in the Cartesian system of reference, $x_{3}=0$ at the still free surface elevation (see Fig. 1). In order to represent the bottom and surface geometry and to correctly assign the pressure and kinematics conditions at the bottom and at the free surface, a particular transformation from Cartesian to curvilinear coordinates, in which coordinates vary in time in order to follow the free surface movements, is introduced:

$$
\xi_{1}=x_{1} \quad \xi_{2}=x_{2} \quad \xi_{3}=\frac{x_{3}+h}{H}
$$

Under the transformation (22) the components of vector $\vec{v}$, are:

$$
v_{1}=0 \quad v_{2}=0 \quad v_{3}=\frac{\partial x_{3}}{\partial t}
$$

This coordinate transformation basically maps the time-varying coordinates of the physical domain into a fixed coordinate system $\left(\xi_{1}, \xi_{2}, \xi_{3}\right)$ where $\xi_{3}$ spans from 0 to 1 . In addition, the Jacobian of the transformation becomes

$$
\sqrt{c}=H
$$

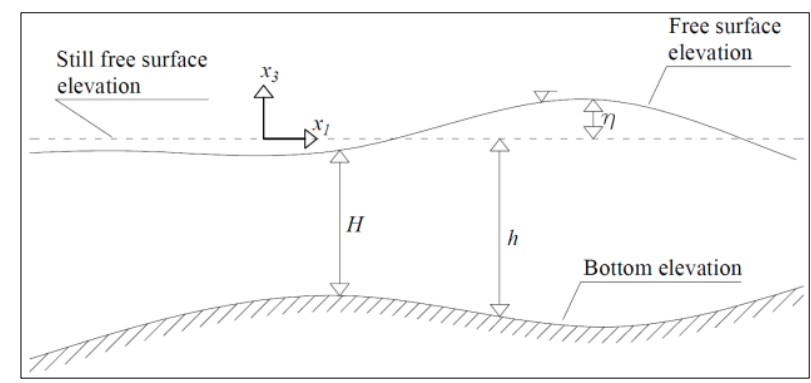

Fig. 1. Definition of the geometric variables. 
We define the cell averaged value (in the transformed space), of the variables $H, H \vec{u}$ and $H a$, respectively (recalling that $H$ does not depend on $\left.\xi_{3}\right)$ :

$$
\begin{gathered}
\bar{H}=\frac{1}{\Delta A_{12}^{*}} \int_{\Delta A_{12}^{*}} H d \xi_{1} d \xi_{2} \\
\overline{H \vec{u}}=\frac{1}{\Delta V^{*}} \int_{\Delta V^{*}} H \vec{u} d \xi_{1} d \xi_{2} d \xi_{3} \\
\overline{H a}=\frac{1}{\Delta V^{*}} \int_{\Delta V^{*}} H a d \xi_{1} d \xi_{2} d \xi_{3}
\end{gathered}
$$

In order to take into account the effects of turbulence, let us split the flow velocity $\vec{u}$, the pressure $p$ and the volume fraction of the solid phase $a$, into a Reynolds-averaged part and a fluctuating part, where the brackets \langle\rangle indicate a Reynolds-average operation and the superscript ' indicates the residual fluctuating part:

$$
\vec{u}=\langle\vec{u}\rangle+\vec{u}^{\prime} \quad p=\langle p\rangle+p^{\prime} \quad a=\langle a\rangle+a^{\prime}
$$

By means of transformation (22), by dividing by $\rho$, eqn. (12) can be rewritten as

$$
\begin{gathered}
\frac{\partial \bar{H}}{\partial t}= \\
-\frac{1}{\Delta A_{12}^{*}} \int_{0}^{1} \sum_{\alpha=1}^{2}\left[\int_{\xi_{\alpha}^{*+}} H(\langle\vec{u}\rangle-\vec{v}) \cdot \vec{c}^{(\alpha)} d \xi_{\beta}\right. \\
\left.-\int_{\xi_{\alpha}^{*-}} H(\langle\vec{u}\rangle-\vec{v}) \cdot \vec{c}^{(\alpha)} d \xi_{\beta}\right]
\end{gathered}
$$

where $\xi_{\alpha}^{*+}$ and $\xi_{\alpha}^{*-}$ are the contour lines of the surface $\Delta A_{12}^{*}$ on which $\xi_{\alpha}$ is constant and which are located at the larger and the smaller value of $\xi_{\alpha}$, respectively (the coefficients $\alpha, \beta=1,2$ are cyclic).

In a Cartesian coordinate system, the particle settling velocity $\vec{w}$ is given by:

$$
w_{1}=0 \quad w_{2}=0 \quad w_{3}=w_{\text {sed }}
$$

in which $w_{\text {sed }}$ is the sediment fall velocity. With the linearization hypothesis, the solid particle velocity is obtained by the superimposition of the fluid velocity field and the particle settling velocity field.
By using of transformation (22), by combining eqns. (13) and (15), we can write the momentum equation under the linearization hypothesis:

$$
\begin{aligned}
& \rho \frac{\partial \overline{H\langle\vec{u}\rangle}}{\partial t}= \\
& -\rho \frac{1}{\Delta V^{*}} \sum_{\alpha=1}^{3}\left\{\int_{\Delta A_{\beta \gamma}^{*+}} H[\langle\vec{u}\rangle \otimes(\langle\vec{u}\rangle-\vec{v})]\right. \\
& \cdot \vec{c}^{(\alpha)} d \xi_{\beta} d \xi_{\gamma} \\
& \left.-\rho \int_{\Delta A_{\beta \gamma}^{*-}} H[\langle\vec{u}\rangle \otimes(\langle\vec{u}\rangle-\vec{v})] \cdot \vec{c}^{(\alpha)} d \xi_{\beta} d \xi_{\gamma}\right\} \\
& +\frac{1}{\Delta V^{*}} \sum_{\alpha=1}^{3}\left[\int_{\Delta A_{\beta \gamma}^{*+}} \mathbf{T} \cdot \vec{c}^{(\alpha)} H d \xi_{\beta} d \xi_{\gamma}\right. \\
& \left.-\int_{\Delta A_{\beta \gamma}^{*-}} \mathbf{T} \cdot \vec{c}^{(\alpha)} H d \xi_{\beta} d \xi_{\gamma}\right] \\
& +\frac{1}{\Delta V^{*}} \sum_{\alpha=1}^{3}\left[\int_{\Delta A_{\beta \gamma}^{*+}} \mathbf{T}_{R} \cdot \vec{c}^{(\alpha)} H d \xi_{\beta} d \xi_{\gamma}\right. \\
& \left.-\int_{\Delta A_{\beta \gamma}^{*-}} \mathbf{T}_{R} \cdot \vec{c}^{(\alpha)} H d \xi_{\beta} d \xi_{\gamma}\right] \\
& +\rho \frac{1}{\Delta V^{*}} \int_{\Delta V^{*}}[1+R\langle a\rangle] \vec{f} H d \xi_{1} d \xi_{2} d \xi_{3}
\end{aligned}
$$

where $R=\breve{\rho}_{S} / \rho-1$ and $\mathbf{T}_{R}=-\rho\left\langle\vec{u}^{\prime} \otimes \vec{u}^{\prime}\right\rangle$ is the turbulent stress tensor. The constitutive relation for the viscous stress tensor is $\mathbf{T}=-\langle p\rangle \mathbf{I}+2 \mu\langle\mathbf{S}\rangle$, where $\mathbf{I}$ is the identity tensor, $\mu$ is the dynamic viscosity and $\langle\mathbf{S}\rangle$ is the rate-of-strain tensor. $v=$ $\mu / \rho$ is the kinematic viscosity. Eqn. (31) can be rewritten as (dividing by $\rho$ ): 


$$
\begin{gathered}
\frac{\partial \overline{H\langle\vec{u}\rangle}}{\partial t}= \\
-\frac{1}{\Delta V^{*}} \sum_{\alpha=1}^{3}\left\{\int_{\Delta A_{\beta \gamma}^{*+}} H[\langle\vec{u}\rangle \otimes(\langle\vec{u}\rangle-\vec{v})]\right. \\
\cdot \vec{c}^{(\alpha)} d \xi_{\beta} d \xi_{\gamma} \\
\left.\int_{\Delta A_{\beta \gamma}^{*-}} H[\langle\vec{u}\rangle \otimes(\langle\vec{u}\rangle-\vec{v})] \cdot \vec{c}^{(\alpha)} d \xi_{\beta} d \xi_{\gamma}\right\} \\
-\frac{1}{\rho} \frac{1}{\Delta V^{*}} \int_{\Delta V^{*}} \frac{\partial\langle p\rangle}{\partial \xi_{k}} \vec{c}^{(k)} H d \xi_{1} d \xi_{2} d \xi_{3} \\
+2 v \frac{1}{\Delta V^{*}} \sum_{\alpha=1}^{3}\left[\int_{\Delta A_{\beta \gamma}^{*+}}\langle\mathbf{S}\rangle \cdot \vec{c}^{(\alpha)} H d \xi_{\beta} d \xi_{\gamma}\right. \\
+\frac{1}{\rho} \frac{1}{\Delta V^{*}} \sum_{\alpha=1}^{3}\left[\int_{\Delta A_{\beta \gamma}^{*+}}\langle\mathbf{T}\rangle \cdot \vec{c}_{R}(\alpha) H d \xi_{\beta} d \xi_{\gamma}\right] \\
+\frac{1}{\Delta V^{*}} \int_{\Delta V^{*}}[1+R\langle a\rangle] \vec{f} H d \xi_{\beta} d \xi_{\gamma} d \xi_{2} d \xi_{3} \\
\left.\mathbf{T}_{R} \cdot \vec{c}^{(\alpha)} H d \xi_{\beta} d \xi_{\gamma}\right]
\end{gathered}
$$

By applying the linearization hypothesis to eqn. (15), by using transformation (22) and by dividing by $\rho$, we can write the particle concentration equation:

$$
\begin{gathered}
\frac{\partial \overline{H\langle a\rangle}}{\partial t}= \\
-\frac{1}{\Delta V^{*}} \sum_{\alpha=1}^{3}\left\{\int_{\Delta A_{\beta \gamma}^{*+}}\langle a\rangle H[(\langle\vec{u}\rangle-\vec{v})-\vec{w}]\right. \\
\cdot \vec{c}^{(\alpha)} d \xi_{\beta} d \xi_{\gamma} \\
\left.-\int_{\Delta A_{\beta \gamma}^{*+}}\langle a\rangle H[(\langle\vec{u}\rangle-\vec{v})-\vec{w}] \cdot \vec{c}^{(\alpha)} d \xi_{\beta} d \xi_{\gamma}\right\} \\
+\frac{1}{\Delta V^{*}} \sum_{\alpha=1}^{3}\left[\int_{\Delta A_{\beta \gamma}^{*+}} H\left\langle\vec{u}^{\prime} a^{\prime}\right\rangle \cdot \vec{c}^{(\alpha)} d \xi_{\beta} d \xi_{\gamma}\right. \\
\left.-\int_{\Delta A_{\beta \gamma}^{*-}} H\left\langle\vec{u}^{\prime} a^{\prime}\right\rangle \cdot \vec{c}^{(\alpha)} d \xi_{\beta} d \xi_{\gamma}\right]
\end{gathered}
$$

Eqns. (29), (32) and (33) represent the formalization of the three-dimensional two-phase flow motion equations under the linearization hypothesis, expressed in integral form in terms of Cartesian based variables, on a time-dependent curvilinear coordinate system.

\subsection{Numerical model}

In this work, eqns. (29), (32) and (33), are numerically solved by adopting the following procedure:

- MUSCL-TVD reconstructions to obtain point-values variables at cell interfaces.

- Advancing in time of the unknown variables at the centre of the cell faces by means of an HLL Riemann solver.

- Advancing in time of the cell-averaged predictor velocity field.

- Solution of the Poisson pressure equation by using a four-color Zebra line Gauss-Seidel alternate method and a multigrid V-cycle.

- Correction of the predictor velocity field by using a scalar potential $\Psi$.

- Advancing in time of the total local depth by means of eqn. (29), and of the volume fraction of the solid phase by means of eqn. (33), by using the corrected velocity field.

To take into account the effects of turbulence, we use a Smagorinsky sub grid model. Further details on the numerical model can be found in [17].

\section{Results and discussion}

The proposed numerical model has been validated against the experimental analysis conducted by Hosseini et al. [21].

The test case geometry consists in a rectangular channel with length $12 \mathrm{~m}$, height $1.5 \mathrm{~m}$ and width $0.75 \mathrm{~m}$. The bottom as a constant slope in the channel length direction. At a channel boundary, inflow conditions are imposed. Three different simulations where reproduced. The inflow conditions and the data of the simulations are reported in table 1 .

Table. 1. Experimental tests conducted by Hosseini et al. [21]. Validation tests parameters.

\begin{tabular}{|l|c|c|c|}
\hline \multicolumn{1}{|c|}{ Test } & A & B & C \\
\hline Solid particle specific gravity $\breve{\rho}_{S} / \rho$ & 2.65 & 2.65 & 2.65 \\
\hline Solid particle diameter $d[\mathrm{~mm}]$ & 0.02 & 0.02 & 0.02 \\
\hline Bottom slope & $3 \%$ & $2 \%$ & $2 \%$ \\
\hline Inflow concentration $\left[\mathrm{Kg} / \mathrm{dm}^{3}\right]$ & 0.01 & 0.00 & 0.01 \\
\hline Liquid flow rate $[\mathrm{l} / \mathrm{min}]$ & 3 & 15 & 10 \\
\hline Inflow velocity $[\mathrm{m} / \mathrm{s}]$ & 0.05 & 0.25 & 0.16 \\
\hline
\end{tabular}



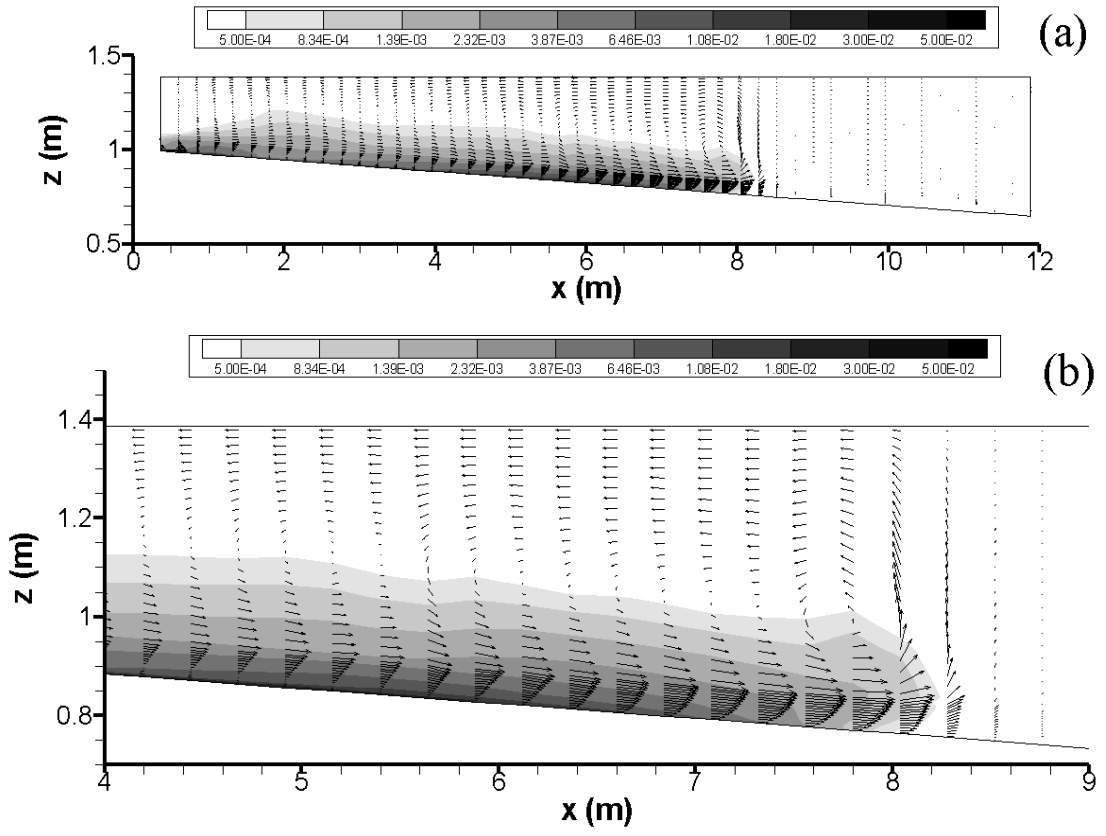

Fig. 2. Numerical simulation by means of the proposed model of the turbidity current of test A (own calculations). a) vertical section of the velocity and concentration fields obtained by means of the proposed simulation model; b) detail of the velocity and concentration fields.

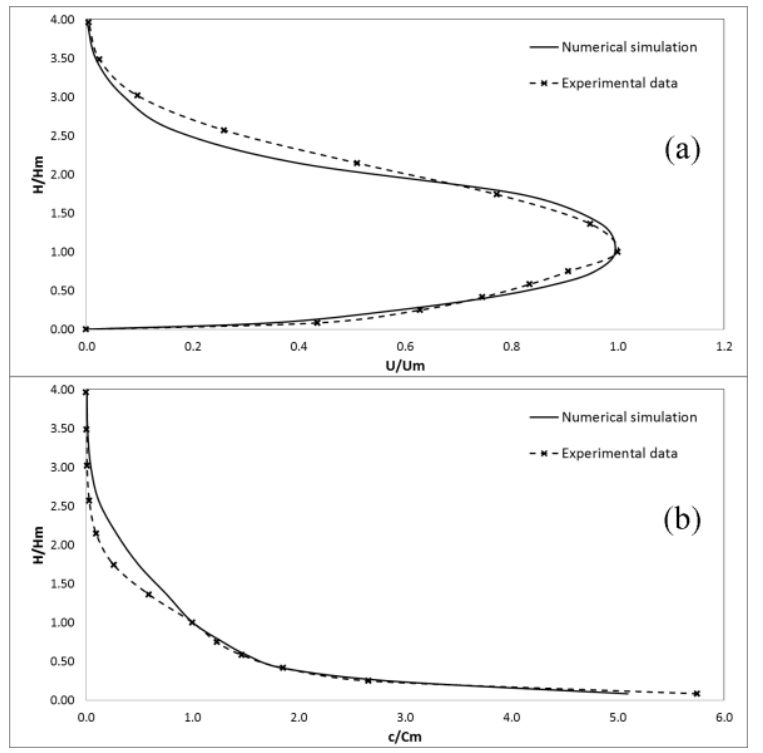

Fig. 3. Comparison between numerical simulation by the proposed model (own calculation, solid line) and experimental results by Hosseini et al. [21] (dashed line) in test A: a) dimensionless velocity profiles; b) dimensionless concentration profiles.

Fig. 2 shows the velocity and concentration fields for the simulation $\mathrm{A}$, at time $t=270 \mathrm{~s}$ from the start of the release of fresh water and solid material.

Fig. 3 shows the comparison between numerical results and experimental data produced by [21] for test $\mathrm{A}$, in terms of mean non-dimensional downstream velocity values (Fig. 3a) and of non- dimensional mean concentration profile (Fig. 3b). The mean non-dimensional velocity is the ratio between the mean velocity and the velocity maximum $U_{m}$; the non-dimensional length scale is the ratio between the thickness of the current $H$ and the height of the velocity maximum, $H_{m}$; the mean non-dimensional concentration is the ratio between the mean concentration and the by the mean concentration at the height of the velocity maximum, $C_{m}$ (see [21]). Analogously, in Fig. 4 and Fig. 5, the comparison between numerical results and experimental data for test $\mathrm{B}$ and test $\mathrm{C}$, respectively, is shown. Figs. 3a, 4a and 5a show that the velocity profiles simulated by the proposed model fit well with the experimental data; the simulated non-dimensional velocity peak values are in very good accordance with the experimental data. With high non-dimensional length, the proposed model underestimates the non-dimensional velocity in test A (Fig. 3a), while slightly overestimates it in test B (Fig. 4a) and C (Fig. 5a). Figs. 3b, 4b and 5b show that there is a good accordance between the concentration profiles simulated by the proposed model and the profiles that results from the experimental data. The proposed model slightly overestimates the non-dimensional mean concentration for high non-dimensional lengths, with respect to the experimental results. 


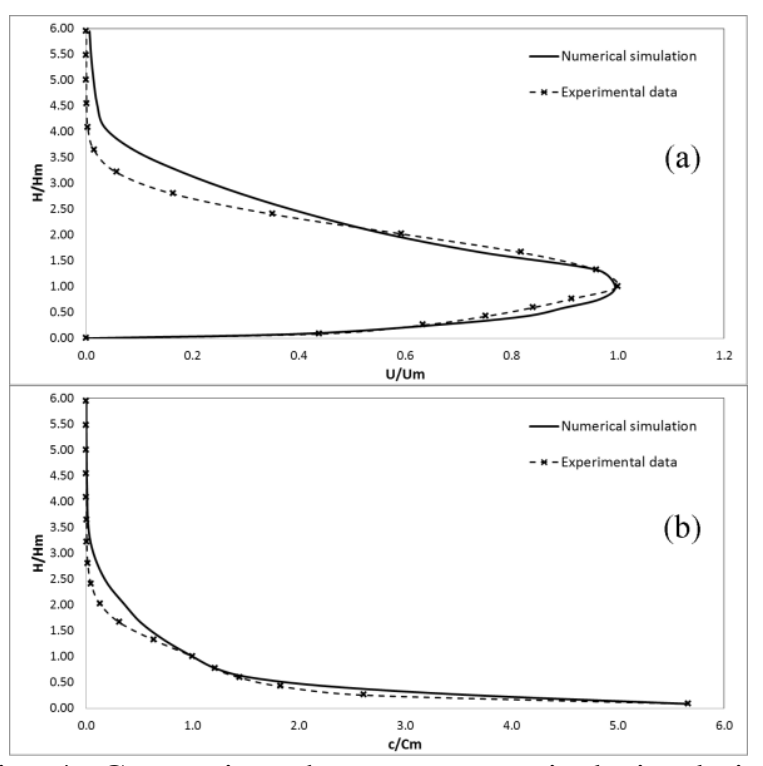

Fig. 4. Comparison between numerical simulation by the proposed model (own calculation, solid line) and experimental results by Hosseini et al. [21] (dashed line) in test $\mathrm{B}$ : a) dimensionless velocity profiles; b) dimensionless concentration profiles.

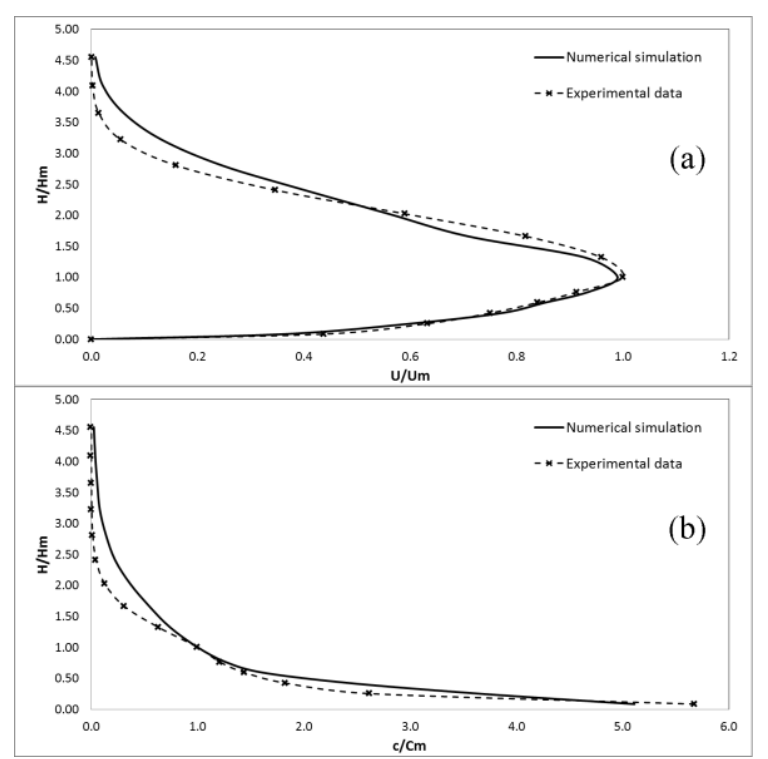

Fig. 5. Comparison between numerical simulation by the proposed model (own calculation, solid line) and experimental results by Hosseini et al. [21] (dashed line) in test $\mathrm{C}$ : a) dimensionless velocity profiles; b) dimensionless concentration profiles.

The proposed numerical model is applied to the case study of the Pieve di Cadore reservoir (Italy). The study is carried out in full reservoir conditions and in liquid and solid inflow discharge conditions associated with flood events with a return period of less than 10 years. The dam bottom outlet is assumed to be open, such as to ensure the complete release of the flood through the bottom outlet. The aim of this case study is to verify the conditions of formation of a turbidity current and its features. This is of a great practical importance, in order to verify in what conditions is useful to use the bottom outlet of a dam to control the silting processes in the reservoir.

Test $\mathrm{D}$ consists in the simulation of a flood event with a return period equal to 2 years. The conditions of the test are: solid particle specific gravity $\breve{\rho}_{S} / \rho=$ 2.65; particle diameter $d=0.1 \mathrm{~mm}$; inflow average concentration of suspended solid $C=5 \mathrm{~g} / \mathrm{l}$; flood peak value of the tributary $Q=100 \mathrm{~m}^{3} / \mathrm{s}$. Fig. 6 shows the velocity and concentration fields in the Pieve di Cadore reservoir for test D. It can be seen that with such conditions, a turbidity current arises. Due to a high bottom slope of the reservoir, the turbidity current reaches the bottom outlet approximately at $t=110 \mathrm{~min}$ from the beginning of the flood.

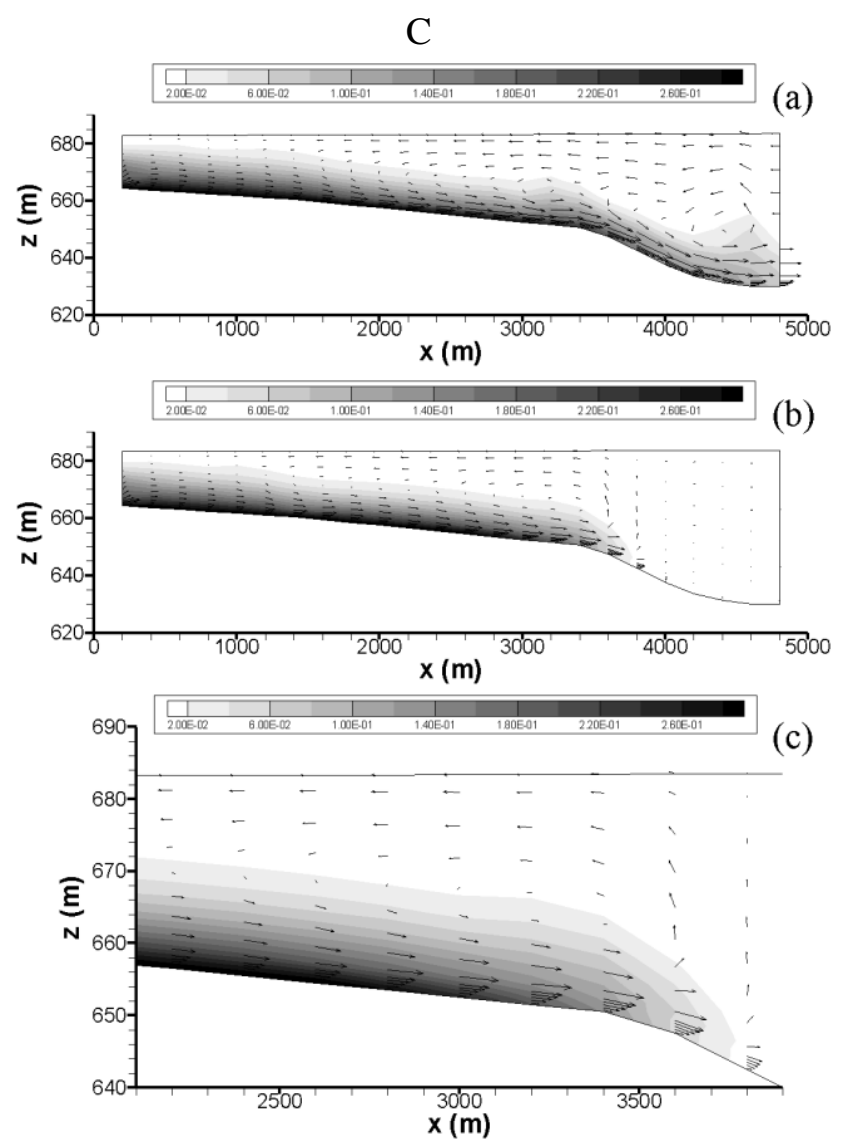

Fig. 6. Test D. Simulation carried out by the proposed model (own calculation). Vertical section of the velocity and concentration fields. a) $t=$ $110 \mathrm{~min}$ from the beginning of the flood; b) $t=$ $95 \mathrm{~min}$ from the beginning of the flood; c) $t=$ $95 \mathrm{~min}$ from the beginning of the flood, detail.

Test $\mathrm{E}$ consists in the simulation of a flood event with a return period of less than 10 years (the inflow average concentration of suspended solid and the 
peak value of the tributary for a return period of 10 years are, respectively, $C=20 \mathrm{~g} / \mathrm{l}$ and $Q=$ $250 \mathrm{~m}^{3} / \mathrm{s}$ ). The conditions of the test are: solid particle specific gravity $\breve{\rho}_{S} / \rho=2.65$; particle diameter $d=0.1 \mathrm{~mm}$; inflow average concentration of suspended solid $C=15 \mathrm{~g} / \mathrm{l}$; flood peak value of the tributary $Q=200 \mathrm{~m}^{3} / \mathrm{s}$. Fig. 6 shows the velocity and concentration fields in the Pieve di Cadore reservoir for test $\mathrm{E}$. As for test $\mathrm{D}$, it can be noted from Fig. 7 that under conditions of test E, a turbidity current is generated and it is able to reach the bottom outlet approximately at $t=90 \mathrm{~min}$ from the beginning of the flood.

In test $\mathrm{F}$, the same flood event described for test $\mathrm{E}$, is reproduced, with the only change in the particle diameter, which is set to $d=0.3 \mathrm{~mm}$. In Fig. 8, the velocity and concentration field are shown for test F. From the comparison between Fig. 7 and Fig. 8 it can be seen that the turbidity current is generated even with a coarser solid material, even if the height of the current substantially decreases with the increase of the particle diameter.

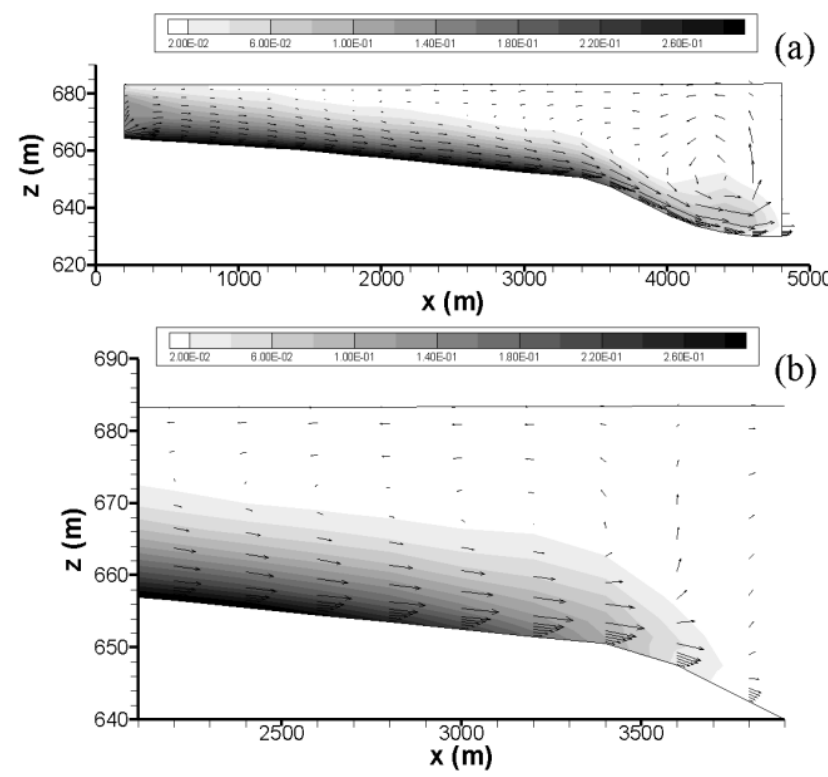

Fig. 7. Test E. Simulation carried out by the proposed model (own calculation). Vertical section of the velocity and concentration fields. a) $t=$ $90 \mathrm{~min}$ from the beginning of the flood; b) $t=$ $70 \mathrm{~min}$ from the beginning of the flood, detail.

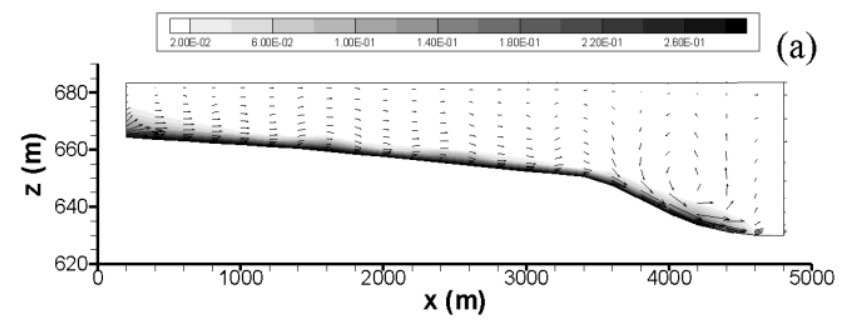

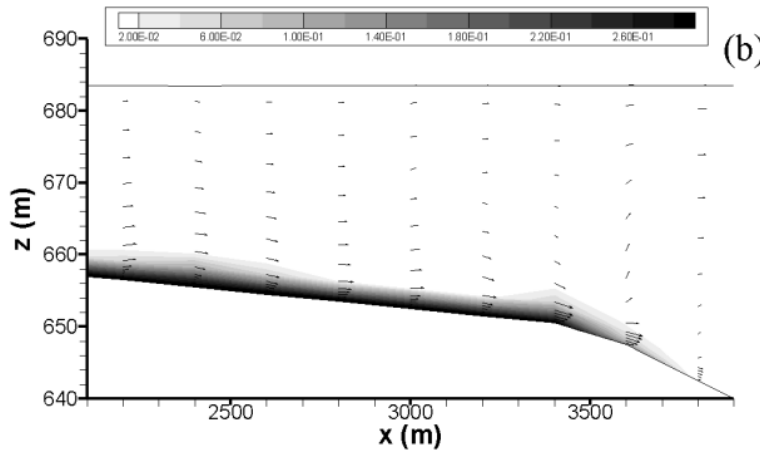

Fig. 8. Test F. Simulation carried out by the proposed model (own calculation). Vertical section of the velocity and concentration fields. a) $t=$ $95 \mathrm{~min}$ from the beginning of the flood; b) $t=$ $80 \mathrm{~min}$ from the beginning of the flood, detail.

\section{Conclusion}

In this work, we proposed a numerical model for turbidity currents, based on two-phase flow motion equations. In particular, we proposed three different formalizations of the two-phase flow motion equations. The most general formalization presented is valid for high concentration values. A more simplified formalization introduces the hypothesis of diluted concentrations. The last formalization presented adopts the linearization hypothesis, i.e. assumes that the particles are in translational equilibrium with the fluid flow. The two-phase flow motion equations are presented in an integral form in time-dependent curvilinear coordinates. The vertical coordinate varies in time in order to follow the free surface movements. The proposed numerical model has been compared with several experimental validation tests. Furthermore, the numerical model has been used to reproduce the case study of Pieve di Cadore reservoir, under several inflow conditions; the possibility of the formation a turbidity current during several different flood events, has been investigated.

\section{References:}

[1] Siddiqui, A.M., Mitkova, M.K. \& Ansari, A.R., Two-phase flow of a third grade fluid between parallel plates, WSEAS Transactions on Fluid Mechanics, Vol. 7(4), 2012, pp. 117-128.

[2] Mitkova, M. K., Siddiqui, A. M., \& Ansari, A. R., Layer Thickness Variation in Two-Phase Flow of a Third Grade Fluid. Applied Mechanics and Materials, Vol. 232, 2012, pp. 273-278.

[3] Abood, S.A., Abdulwahid, M.A. \& Almudhaffar, M.A., Comparison between the 
experimental and numerical study of (air-oil) flow patterns in vertical pipe, Case Studies in Thermal Engineering, Vol. 14, 2019, pp. 1-11.

[4] Abood, S.A., Abdulwahid, M.A. \& Almudhaffar, M.A., Experimental Study of (Water-oil) Flow Patterns and Pressure Drop in Vertical and Horizontal Pipes, International Journal of Air-Conditioning and Refrigeration, Vol. 26 (4), 2018.

[5] Abdulwahid, M.A., Kareem, H.J. \& Almudhaffar, M.A., Numerical Analysis of Two Phase Flow Patterns in Vertical and Horizontal Pipes, WSEAS Transactions on Fluid Mechanics, Vol. 12, 2017, pp. 131-140.

[6] Ungarish, M., Benjamin's gravity current into an ambient fluid with an open surface in a channel of general cross-section, Journal of Fluid Mechanics, Vol. 859, 2019, pp. 972-991.

[7] Longo, S., Ungarish, M., Di Federico, V., Chiapponi, L., Petrolo, D., Gravity currents produced by lock-release: Theory and experiments concerning the effect of a free top in non-Boussinesq systems, Advances in Water Resources, Vol. 121, 2018, , pp. 456-471.

[8] Hogg, A.J., Nasr-Azadani, M.M., Ungarish, M. \& Meiburg, E., Sustained gravity currents in a channel, Journal of Fluid Mechanics, Vol. 798, 2016, pp. 853-888.

[9] Salinas, J.S., Bonometti, T., Ungarish, M. \& Cantero, M.I., Rotating planar gravity currents at moderate Rossby numbers: Fully resolved simulations and shallow-water modeling, Journal of Fluid Mechanics, Vol. 867, 2019, pp. 114-145.

[10] Ungarish, M., An Introduction to Gravity Currents and Intrusions. Chapman \& Hall/CRC Press, 2009.

[11] Espath, L.F.R., Pinto, L.C., Laizet, S. \& Silvestrini, J.H., Two- and three-dimensional Direct Numerical Simulation of particle-laden gravity currents, Computers \& Geosciences, Vol. 63, 2014, pp. 9-16.

[12] Cantero, M.I., Balachandar, S., Cantelli, A., Pirmez, C. \& Parker, G., Turbidity current with a roof: Direct numerical simulation of selfstratified turbulent channel flow driven by suspended sediment, Journal of Geophysical Research: Oceans, Vol. 114. C03008, 2009, pp. 1-20.

[13] Cannata, G., Petrelli, C., Barsi, L., Fratello, F. \& Gallerano, F., A dam-break flood simulation model in curvilinear coordinates, WSEAS Transactions on Fluid Mechanics, Vol. 13, 2018, pp. 60-70.
[14] Cannata, G., Barsi, L., Petrelli, C. \& Gallerano, F., Numerical investigation of wave fields and currents in a coastal engineering case study, WSEAS Transactions on Fluid Mechanics, Vol. 13, 2018, pp. 87-94.

[15] Sørensen, O.R., Schäffer, H.A. \& Sørensen, L.S., Boussinesq-type modelling using unstructured finite element technique. Coastal Engineering, Vol. 50, No. 4, 2003, pp. 181198.

[16] Cannata, G., Petrelli, C., Barsi, L., Camilli, F. \& Gallerano, F., 3D free surface flow simulations based on the integral form of the equations of motion, WSEAS Transactions on Fluid Mechanics, Vol. 12, 2017, pp. 166-175.

[17] Cannata, G., Petrelli, C., Barsi, L. \& Gallerano, F., Numerical integration of the contravariant integral form of the Navier-Stokes equations in time-dependent curvilinear coordinate system for three-dimensional free surface flows, Continuum Mechanics and Thermodynamics, Vol. 31, No. 2, 2019, pp. 491-519.

[18] Cannata, G., Gallerano, F., Palleschi, F., Petrelli, C. \& Barsi, L., Three-dimensional numerical simulation of the velocity fields induced by submerged breakwaters, International Journal of Mechanics, Vol. 13, 2019, pp. 1-14.

[19] Ma, G., Shi, F. \& Kirby, J.T., Shock-capturing non-hydrostatic model for fully dispersive surface wave processes, Ocean Modelling, Vol. 43-44, 2012, pp. 22-35.

[20] S.F., Bradford, Numerical Simulation of Surf Zone Dynamics, Journal of Waterway Port Coastal and Ocean Engineering, Vol. 126, No.1, 2000, pp. 1-13.

[21] Hosseini, S.A., Shamsai, A. \& Ataie-Ashtiani, B., Synchronous measurements of the velocity and concentration in low density turbidity currents using an Acoustic Doppler Velocimeter. Flow Measurement and Instrumentation, Vol. 17, 2006, pp. 59-68.

[22] Thompson, J.F., Warsi, Z.U.A. \& Mastin, C.W., Numerical Grid Generation, ed. NorthHolland: New York, Amsterdam and New York, 1985.

[23] Aris, R., Vectors, tensors, and the basic equations of fluid mechanics, New York, USA, Dover, 1989. 